\title{
Correction to: Contextualism, Factivity and Closure
}

\section{Correction to:}

S. Leardi and N. Vassallo,

Contextualism, Factivity and Closure, SpringerBriefs in Philosophy, https://doi.org/10.1007/978-3-030-16155-2

The subtitle of the book "An Union that Should Not Take Place?" in the original version of the book has been changed to "A Union that Should Not Take Place?"

The correction book has been updated with the change. 